The Chittagong Univ. J. Sci. 42(1): 58-83, 2020

\title{
Thermochemical, Molecular Docking and ADMET Studies of Some Methyl $\alpha$-D-Glucopyranoside Derivatives
}

\author{
Jannatul Ferdous and Sarkar M. A. Kawsar \\ Department of Chemistry, Faculty of Science, University of Chittagong, \\ Chittagong 4331, Bangladesh \\ *Corresponding author; E-mail: akawsarabe@yahoo.com
}

Manuscript received on 08 November, 2020, Revised manuscript received on 20 December, 2020 and accepted on 24 December, 2020.

\begin{abstract}
In this study, methyl $\alpha$-D-glucopyranoside and its modified derivatives were optimized by employing density functional theory (DFT) with B3LYP/3-21G level theory to explore their structural and thermodynamic properties. Electronic energies, enthalpies, Gibbs free energies, dipole moments, Highest occupied molecular orbital (HOMO)-lowest unoccupied molecular orbital (LUMO) gaps, the density of states (DOS) and molecular electrostatic potential (MEP) of these modified compounds were found optimal in the subsequent analysis. Molecular docking has been performed against potential inhibitors of the Bacillus subtilis Obg protein (PDB: 1LNZ) to search the binding affinity and mode(s) of six compounds that have significant antibacterial and antifungal activities. It was found that the selected glucopyranoside derivatives have strong interactions mainly with Arg236 and Arg238 residues of the Bacillus subtilis Obg protein, and the molecules were surrounded by other active site residues like Lys156, Pro91, and Glu333. An absorption, distribution, metabolism, excretion, and toxicity (ADMET) calculation reveals that the modified derivatives are less toxic and have improved pharmacokinetic features over the parent drug. This computational investigation showed that these methyl $\alpha$-D-glucopyranoside derivatives might be used as potential inhibitors against the promising antibacterial and antifungal activities for future studies.
\end{abstract}

Keywords: Monosaccharide, Bacillus subtilis, HOMO-LUMO, DFT, ADMET

DOI: https://doi.org/10.3329/cujs.v42i1.54238 
এ গবেষণায়, মিথাইল $\alpha$-ডি-গুরোপাইরানোসাইড এবং এর কিছু অভিশ্রুতি জাতকের কাঠামোগত এবং তাপগতীয় বৈশিষ্ট্য অন্বেষণ DFT-B3LYP/3-21G করতে স্তরের ঘনত্ব ক্রিয়ামূলক তত্ত্ব (DFT) প্রয়োগ করে অনুকূলিত করা হয়েছে। পরবর্তী বিশ্লেষণে, বৈদ্যুতিক শক্তিসমূহ, এনথ্যালপিসমূহ, গিবসের মুক্ত শক্তিসমূহ, ডাইপোল মোন্নেন্ট, HOMO-LUMO শক্তি, অবস্থার ঘনত্ব (DOS) এবং আণবিক বৈদ্যুতিক সম্ভাবনা (MEP) অনুসন্ধান করা হয়েছে। Bacillus subtilis Obg প্রোটিন (PDB: 1LNZ) এর সম্ভাব্য বাধাদায়ক খুঁজে পেতে ছয়টি যৌগ যাদের উল্লেখযোগ্য অ্যান্টিব্যাকটেরিয়াল এবং অ্যান্টিফাঙ্গাল ক্রিয়াকলাপ আছে তাদের বিপরীতে আণবিক ডকিং সম্পন্ন করা হয়েছে, যাতে তাদের বন্ধন প্রবণতা এবং ধরন(গুনো) অনুসন্ধান করা যায়। দেখা গেছে যে, নির্বাচিত গ্লুকোপাইরানোসাইড জাতকসমূহ প্রধানত: Bacillus subtilis Obg প্রোটিনের Arg236 এবং Arg238 অবশিষ্টাংশসমূহের সাথে শক্তভাবে মিথস্ক্রিয়া করেছে এবং অন্যান্য সক্রিয় অংশের অবশিষ্টাংশ যেমন, Lys156, Pro91 এবং Glu333 অণুসমূহকে চারপাশে ঘিরে রেখেছে। ADMET হিসাব উদঘাটন করে যে, অভ্রির্যুতি জাতকসমূহ কম বিষাক্ত এবং জনক ভেষজের তুলনায় তাদের উন্নত ফার্মাকোকাইনেটিক বৈশিষ্ট্য রয়েছে। এই কম্পিউটেশনাল অনুসন্ধানে দেখা গেছে যে, এই মিথাইল $\alpha$-ডি-গুরোপাইরানোসাইড জাতকসমূহ সম্ভাব্য বাধাদায়ক এবং আশাপ্রদ অ্যান্টিব্যাকটেরিয়াল এবং অ্যান্টিফাঙ্গাল ক্রিয়াকলাপের জন্য ভবিষ্যৎ গবেষণায় ব্যবহৃত হতে পারে।

\section{Introduction}

Carbohydrates are the most abundant and the most diverse biopolymers in nature and also key molecules in nature with several roles in biological processes. For a long time, carbohydrates have been a very attractive topic for scientists due to their immense importance in biological systems including viral and bacterial infections, cell growth and proliferation, cell-cell communication as well as an immune response [1, 2]. They are the source of the metabolic energy supply, but also for the fine-tuning of cell-cell interactions and other crucial processes [3, 4]. It was found from the literature survey that a large number of biologically active compounds contain aromatic, heteroaromatic and acyl substituents [5]. The benzene substituted benzene and also nitrogen, sulphur and halogen containing substituents are also known to enhance the biological activity of the parent compound [6]. It is also known that if an active nucleus is linked to another active nucleus, the resulting molecule may possess greater potential for biological activity [5]. Moreover, selective acylation of carbohydrates [7, 8] and also evaluation of microbial activities [9-11] revealed that in many cases the combination of two or more 
60 Thermochemical, Molecular Docking and ADMET Studies of Some Methyl $\alpha$-D-Glucopyranoside Derivatives

heteroaromatic nuclei and acyl groups enhances the biological activity manifold than its parent nucleus [12-14].

In a recent study, some monosaccharide derivatives are found as a potential inhibitor against cancer cell protein 4ZZZ [15]. Encouraged by reports [15-17], the present study attempted to optimize modified methyl $\alpha$-D-glucopyranoside (Figure 1) derivatives to explore their thermal, electrical stability and biochemical behavior based on quantum mechanical methods. The free energy, enthalpy, entropy, dipolemoment, HOMO-LUMO gap, DOS plot, and molecular electrostatic potential were calculated to compare their thermal and chemical characteristic. It was revealed that the HOMO-LUMO gap of the compounds $(\mathbf{4}, \mathbf{6}, \mathbf{7}$ and 8) having long acyl chains were higher than the compounds $(\mathbf{9}, \mathbf{1 0}, 11$ and 12) bearing halogenated aromatic ring, which also indicated their fair reactivity. Further, some selected derivatives (having better antibacterial and antifungal activity) were employed for molecular docking against Bacillus subtilis Obg (PDB: 1LNZ) receptor protein to understand their nonbonding interactions, binding mode, and binding affinity. Obg proteins are among a large group of GTP binding proteins conserved from bacteria to man [18]. Finally, pharmacokinetic enumeration was investigated to compare their absorption, metabolism and toxicity and understand the thermodynamic, molecular orbital, molecular electrostatic potential, and ADMET properties of methyl $\alpha$-Dglucopyranoside and its derivatives for the first time.

A



$\mathrm{B}$

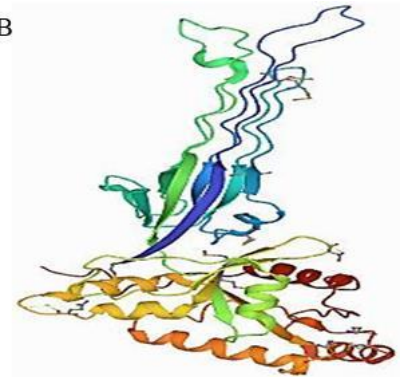

Figure 1. Structure of the methyl $\alpha$-D-glucopyranoside(A) and crystal structure of the $1 \mathrm{LNZ}(\mathrm{B})$. 


\section{Computational methods}

\subsection{Designing and optimization of methyl $\alpha$-D-glucopyranoside derivatives}

In computational chemistry, quantum mechanical methods are widely used to calculate thermal, molecular orbitals and molecular electrostatic properties [19]. Geometry optimization and further modification of all synthesized derivatives were carried out using the Gaussian 09 program [20]. Density functional theory (DFT) with Beck's (B) [21] three-parameter hybrid model, and Lee, Yang and Parr's (LYP) [22] correlation functional under 3-21G basis set was employed to optimize and predict their thermal and molecular orbital properties. Dipole moment, enthalpy, free energy and entropy were calculated for all the compounds. Frontier molecular orbital features HOMO (highest occupied molecular orbital), LUMO (lowest unoccupied molecular orbital) were counted at the same level of theory. For each of the methyl $\alpha$-D-glucopyranoside derivative, HOMO-LUMO energy gap, hardness $(\eta)$, and softness $(S)$ were calculated from the energies of frontier HOMO and LUMO according to Parr and Pearson's interpretation of DFT and Koopmans theorem [23] on the correlation of ionization potential (I) and electron affinities (E) with HOMO and LUMO energies $(\varepsilon)$. The following equation was used to calculate hardness $(\eta)$, softness $(S)$.

$$
\eta=\frac{[\varepsilon \text { LUMO }-\varepsilon \text { HOMO }]}{2} ; \quad S=\frac{1}{\eta}
$$

\subsection{Protein designing and molecular docking}

The three-dimensional structure of Bacillus subtilis Obg(PDB ID. 1LNZ) was retrieved in pdb format from the protein data bank [24]. All heteroatoms and water molecules were removed by using PyMol (version 1.3) software packages [25]. Swiss-Pdb viewer software (version 4.1.0) was employed for energy minimization of the protein [26]. Then optimized drugs were subjected for molecular docking study against bacterial protein Bacillus subtilis $\mathrm{Obg}(\mathrm{PDB}$ ID. 1LNZ). In fine, molecular docking simulation was rendered by PyRx software (version 0.8) [27] considering the protein as a macromolecule and the drug as a ligand. AutodockVina was employed for docking analysis, and AutoDock Tools (ADT) of the MGL 
software package was used to convert pdb into a pdbqt format to input protein and ligands. The size of the grid box in AutoDockVina was kept at 56.9266, 110.4821, and $45.0085 \AA$ for X, Y, Z directions, respectively. After completion of docking, both the macromolecule and ligand structures were saved in. pdbqt format as needed by Accelrys Discovery Studio (version 4.1) to explore and visualize the docking result and search the non-bonding interactions between ligands and amino acid residues of receptor protein [28].

\subsection{ADMET study}

The admetSAR server was to study the pharmacokinetic parameters and toxicity of the modified methyl $\alpha$-D-glucopyranoside derivatives and parent compound. We used the admetSAR online database to calculate the pharmacokinetic parameters related to drug absorption, metabolism and toxicity of the parent drug and its designed derivatives [28]. Using structure similarity search methods, admetSAR predicts the latest and most comprehensive, manually curated data for diverse chemicals associated with known ADMET profiles. Generally, drug-likeness is evaluated using Lipinski's rule of five [29]. Although it is quite difficult to verify all of these compounds and to know whether this program included organic-based drugs or not, well known Pt-based cisplatin and carboplatin as well as metal-based drugs approved by the Food and Drug Administration (FDA) and in clinical trials were used test candidates to verify the glucopyranoside derivatives.

\subsection{Strategies and optimization of designed methyl $\alpha$-D-glucopyranoside derivatives}

Methyl $\alpha$-D-glucopyranoside derivatives used in this study were designated and optimized in quantum mechanical method according to the reaction scheme (Figure 2) [30]. 

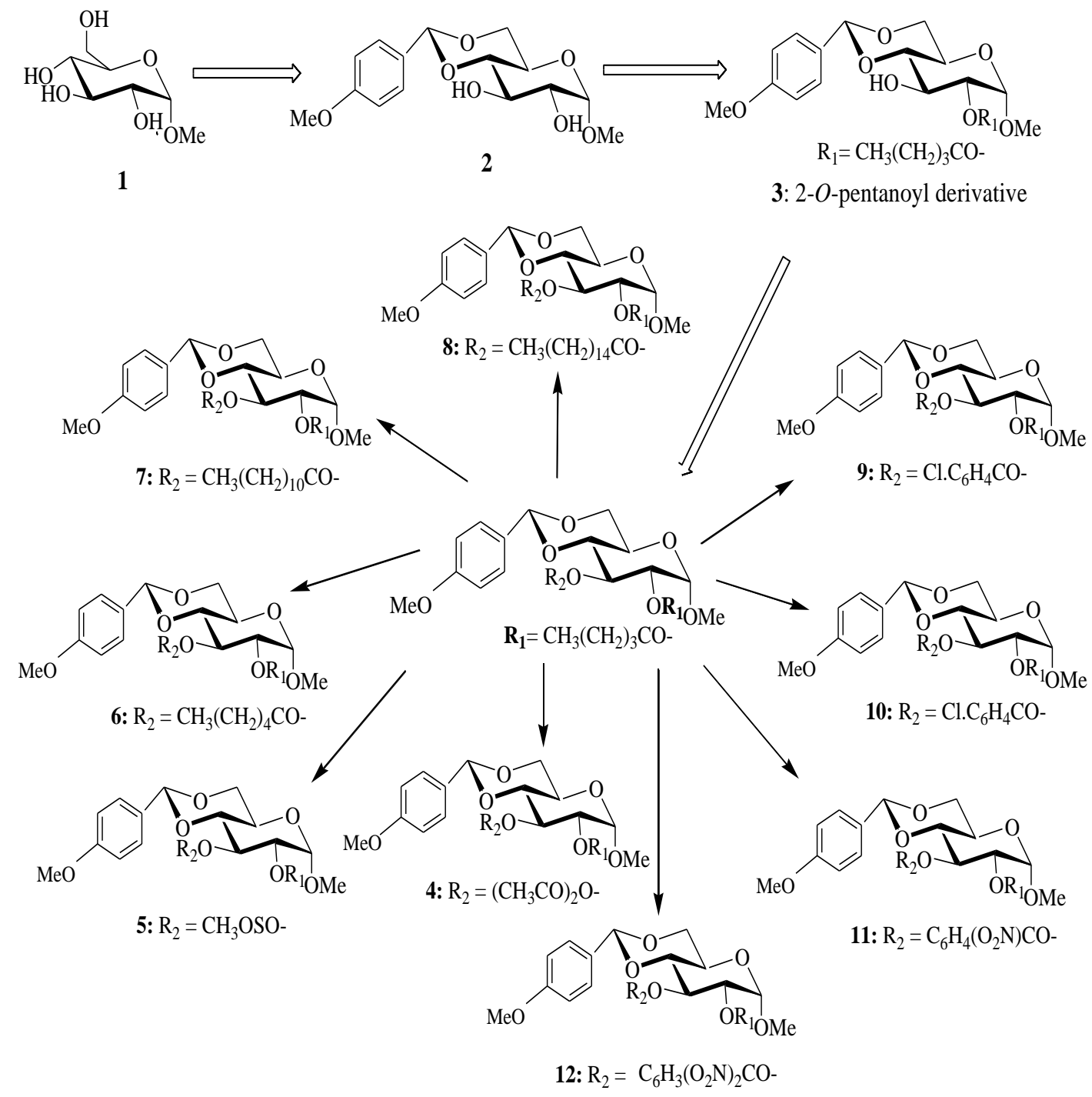

Figure 2. Chemical structure of the methyl $\alpha$-D-glucopyranoside derivatives (2-12). 
64 Thermochemical, Molecular Docking and ADMET Studies of Some Methyl $\alpha$-D-Glucopyranoside Derivatives

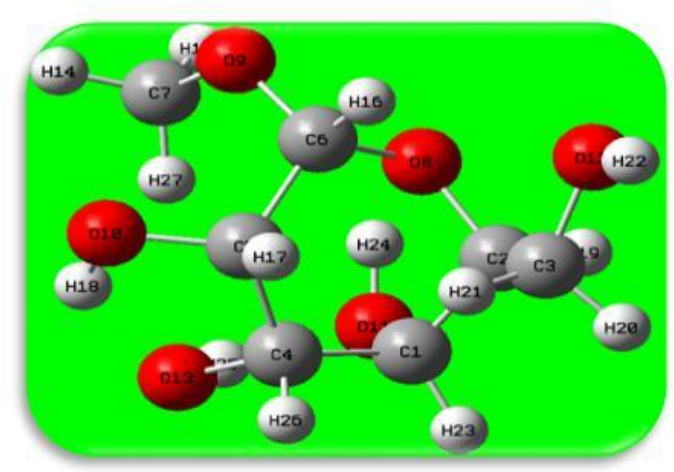

Methyl $\alpha$-D-galactopyranoside (1)

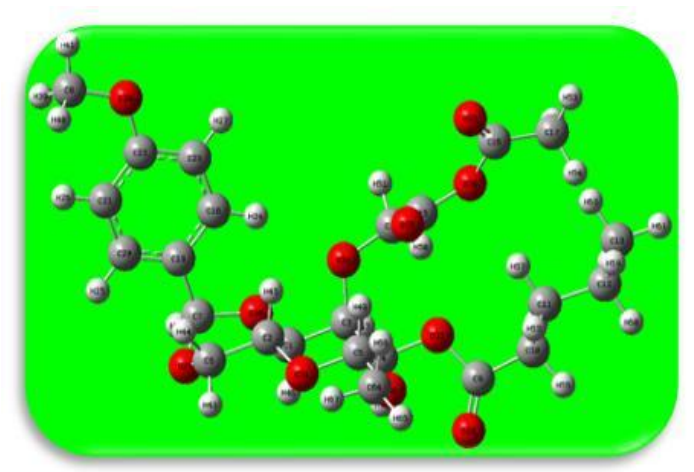

Compound (4)

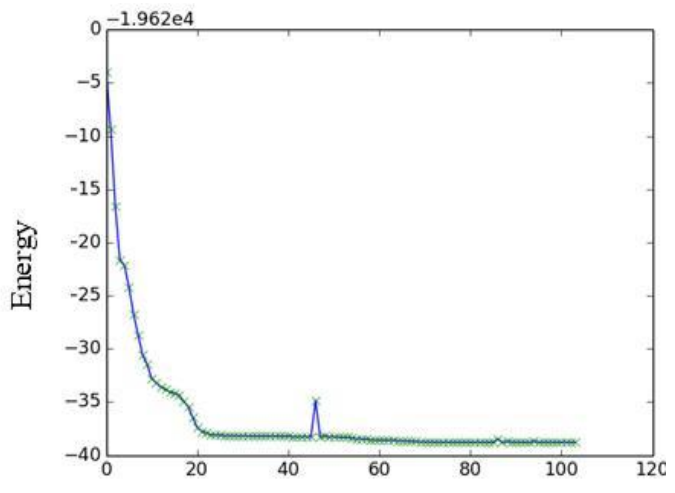

Optimization step

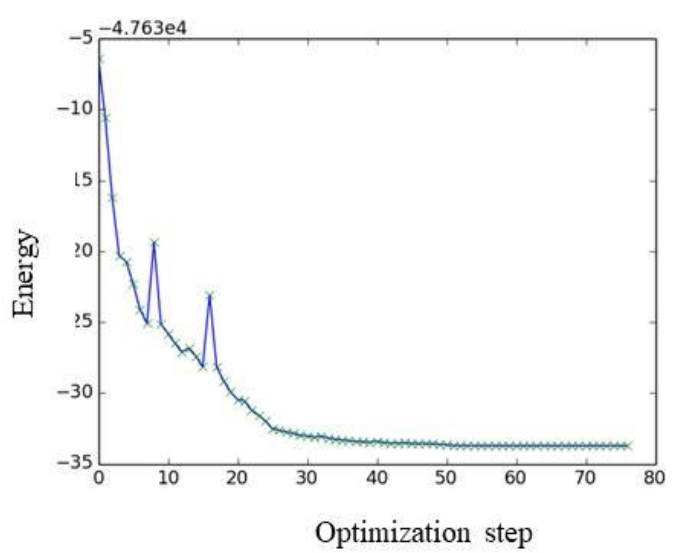

Continued.... 


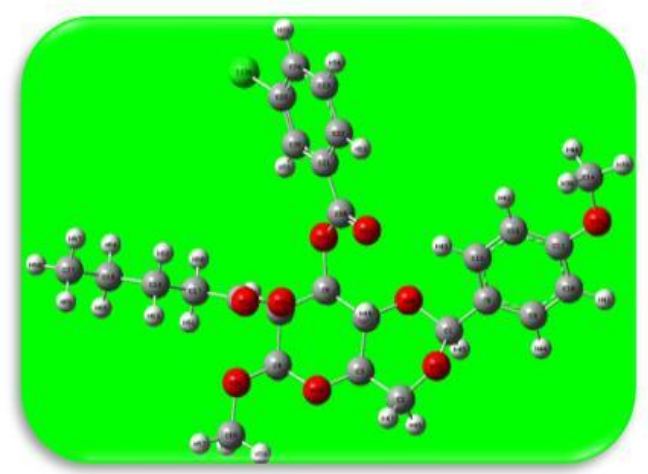

Compound (9)

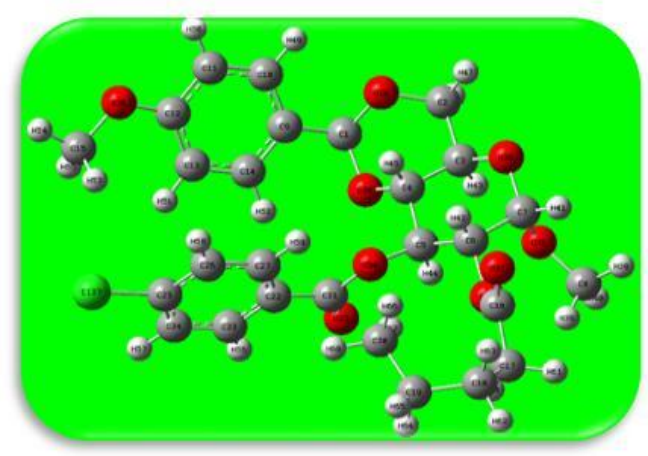

Compound (10)
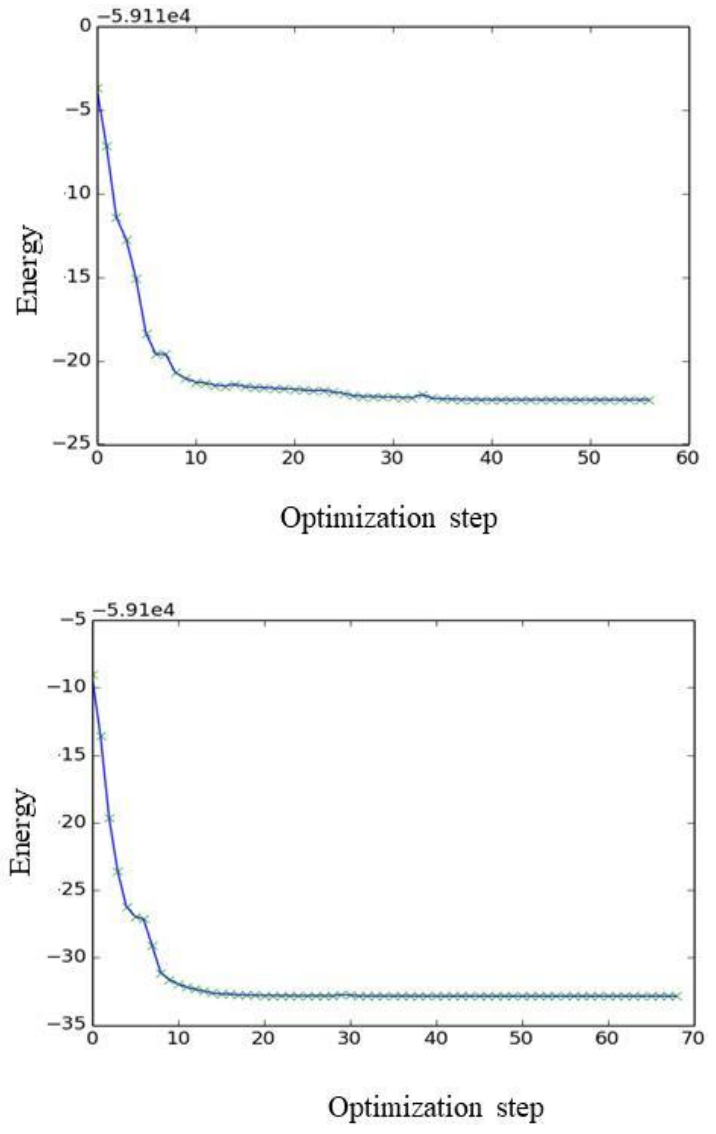

Continued.... 
66 Thermochemical, Molecular Docking and ADMET Studies of Some Methyl $\alpha$-D-Glucopyranoside Derivatives

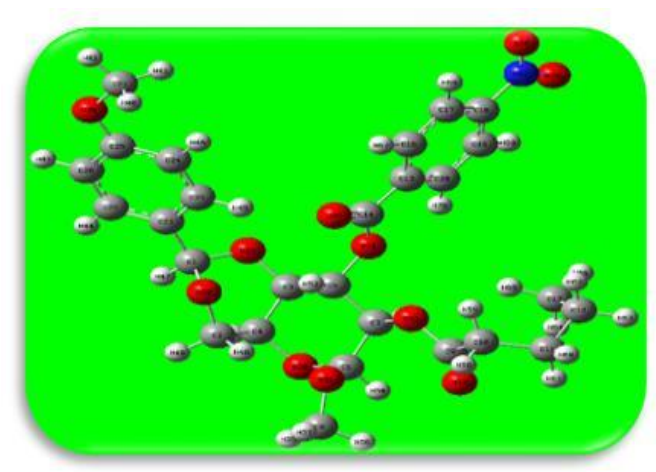

Compound (11)

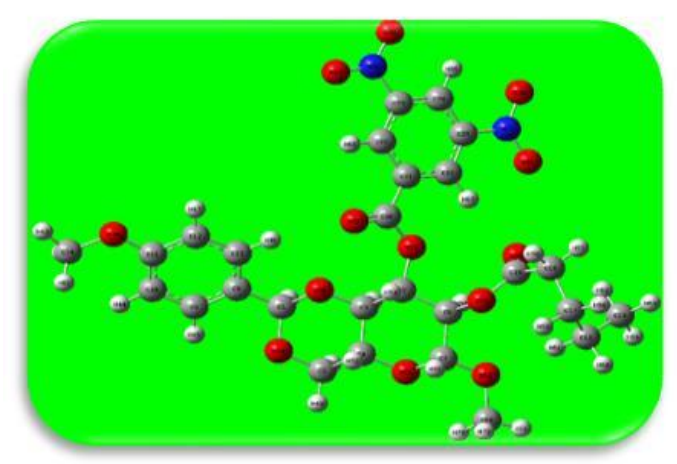

Compound (12)
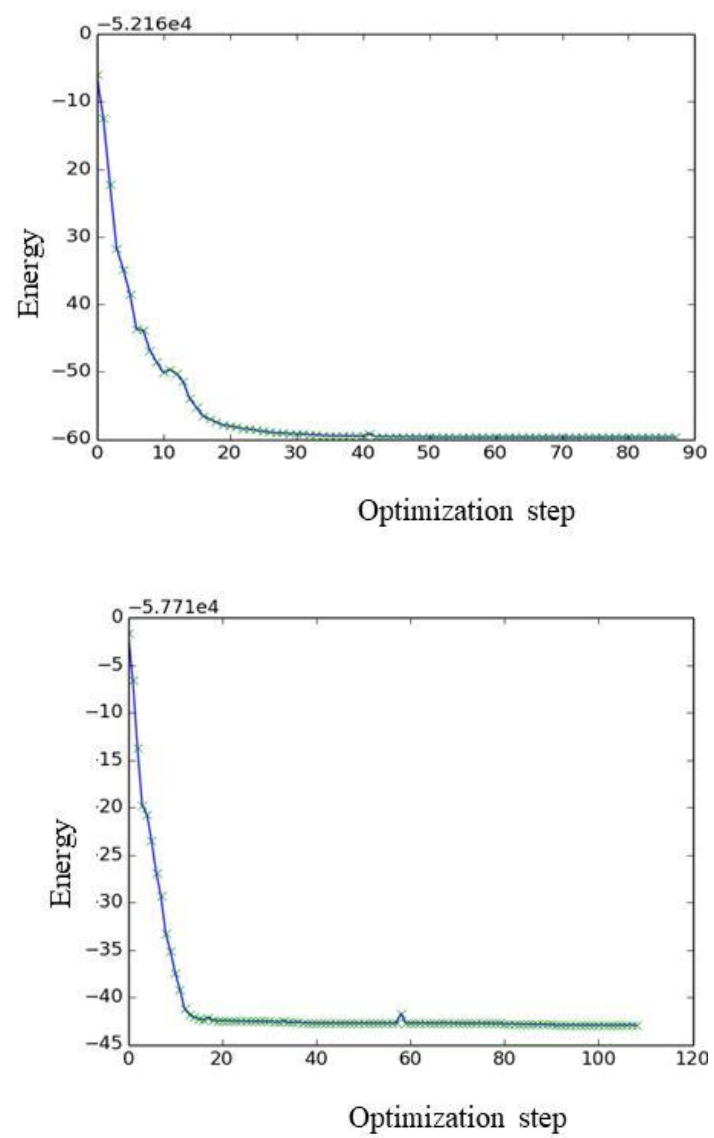

Figure 3. Stable optimized structure and geometrical optimization curve methyl $\alpha$ D-glucopyranoside (1) and some of its derivatives $(4,9,10,11$ and 12).

In computational chemistry, energy minimization is the process to identify an arrangement in space of a collection of atoms where, according to some computational level of chemical bonding, the net inter-atomic force on each atom is acceptably close to zero and the position on the potential energy surface (PES) is a stationary point. Geometry optimization is the physical significance of the obtained structure: optimized structures often correspond to a substance as it is found in 
nature and the geometry of such a structure can be used in a variety of experimental and theoretical investigations in the fields of chemical structure, thermodynamics, chemical kinetics, spectroscopy and others. The lowest energy conformation could be calculated from the bond lengths and angles with the smallest steric energy. By this, we mean finding a point in configuration space where all of the forces on the atoms are balanced. By simply minimizing the energy of a molecule, we are able to identify the stable conformations.

The energy minimization curve of the above compounds showed that energy minimization occurred more smoothly in the case of derivatives than the parent compound 1 (Figure 3). Besides compounds (9 and 10), showed the highest minimized energy, the curve is straightly coming down to the ground state. Comparatively, a lower deviation is observed for the derivatives than the parent drug. Compound 4 showed a vastly zigzag deviation curve while the other derivatives showed a smooth curve. So, it is determined that the synthesized compounds were stable nearly to the parent compound, methyl $\alpha$-Dglucopyranoside (1).

\section{Results and Discussion}

\subsection{Thermodynamic study}

A simple modification of chemical structure significantly influences the structural properties including thermal and molecular orbital parameters. The spontaneity of a reaction and stability of a product can be predicted from the free energy and enthalpy values [31]. Highly negative values are more suitable for thermal stability. In drug designing, hydrogen bond formation and non-bonded interactions are influenced by dipole moment. A comparatively higher dipole moment can improve the binding property [32] of a molecule. The highest free energies were -2172.610 (Hartree) and -2172.593 (Hartree) observed for two derivatives (9 and 10) and which also showed the highest enthalpies -2172.484 (Hartree) and -2172.503 (Hartree) and highest electronic energies -2172.485 (Hartree) and -2172.504 (Hartree). The highest dipole moment was 7.827 (Debye) observed for derivative 
12, whereas derivative 5 exhibited the lowest value 1.736 (Debye) (Table-1). These values were gradually increased with longer carbon chains (1-8) and halogenated derivatives (9-12) (Table 1). However, halogenated and aromatic derivatives exhibited better free energy and dipole moment, as recorded by compounds 9 (2-Cl.Bz), 10 (4-Cl.Bz), $11\left(4-\mathrm{NO}_{2} . \mathrm{Bz}\right)$ and $12\left(3,5-\mathrm{di}-\mathrm{NO}_{2} . \mathrm{Bz}\right)$, which had the highest free energy of the therapeutics under investigation and showed markedly improved dipole moment. Therefore, this discourse proved that modification of hydroxyl (-OH) groups of methyl $\alpha$-D-glucopyranoside significantly increased its thermodynamic properties which indicated the inherent stability of the synthesized derivatives [15-17]. It was observed that, among all the synthesized derivatives, compound 8 comprisethe highest molecular weight $(634.85 \mathrm{~g} / \mathrm{mol})$ showed the maximum thermodynamic values even acyl chain containing derivatives (2-8).

Table 1. The stoichiometry, molecular weight ( $\mathrm{g} / \mathrm{mol})$,electronic energy, enthalpy, Gibbs free energy (Hartree) and dipole moment (Debye) of methyl $\alpha$-Dglucopyranoside and its derivatives.

\begin{tabular}{ccccccc}
\hline Entry & Stoichiometry & $\begin{array}{c}\text { Molecular } \\
\text { weight }\end{array}$ & $\begin{array}{c}\text { Electronic } \\
\text { energy }\end{array}$ & Enthalpy & $\begin{array}{c}\text { Gibbs free } \\
\text { energy }\end{array}$ & $\begin{array}{c}\text { Dipole } \\
\text { moment }\end{array}$ \\
\hline 1 & $\mathrm{C}_{7} \mathrm{H}_{14} \mathrm{O}_{6}$ & 194.18 & -722.209 & -722.2084 & -722.2608 & 4.771 \\
2 & $\mathrm{C}_{14} \mathrm{H}_{19} \mathrm{O}_{7}$ & 312.32 & -1103.683 & -1103.682 & -1103.752 & 3.692 \\
3 & $\mathrm{C}_{19} \mathrm{H}_{27} \mathrm{O}_{8}$ & 396.44 & -1372.686 & -1372.685 & -1372.776 & 2.026 \\
4 & $\mathrm{C}_{21} \mathrm{H}_{29} \mathrm{O}_{9}$ & 496.51 & -1751.022 & -1751.021 & -1751.128 & 3.494 \\
5 & $\mathrm{C}_{20} \mathrm{H}_{29} \mathrm{SO}_{9}$ & 474.53 & -1957.423 & -1957.422 & -1957.522 & 1.736 \\
6 & $\mathrm{C}_{25} \mathrm{H}_{37} \mathrm{O}_{9}$ & 494.58 & -1680.745 & -1680.744 & -1680.856 & 1.624 \\
7 & $\mathrm{C}_{27} \mathrm{H}_{41} \mathrm{O}_{9}$ & 578.14 & -1915.165 & -1915.164 & -1915.293 & 4.135 \\
8 & $\mathrm{C}_{35} \mathrm{H}_{57} \mathrm{O}_{9}$ & 634.85 & -2071.459 & -2071.458 & -2071.591 & 2.322 \\
9 & $\mathrm{C}_{26} \mathrm{H}_{30} \mathrm{O}_{9} \mathrm{Cl}$ & 534.99 & -2172.485 & -2172.484 & -2172.593 & 3.791 \\
10 & $\mathrm{C}_{26} \mathrm{H}_{30} \mathrm{O}_{9} \mathrm{Cl}$ & 534.99 & -2172.504 & -2172.503 & -2172.610 & 3.359 \\
11 & $\mathrm{C}_{26} \mathrm{H}_{30} \mathrm{O}_{11} \mathrm{~N}$ & 545.54 & -1918.437 & -1918.436 & -1918.545 & 4.411 \\
12 & $\mathrm{C}_{26} \mathrm{H}_{29} \mathrm{O}_{13} \mathrm{~N}_{2}$ & 590.54 & -2121.776 & -2121.776 & -2121.891 & 7.827 \\
\hline
\end{tabular}


Besides, halogenated derivatives (9-12) were also increased their properties with the increase of molecular weight. These analyses evolved that the increments of the molecular weight of methyl $\alpha$-D-glucopyranoside derivatives improve their stability.

\subsection{Frontier molecular orbitals analysis}

The frontier molecular orbitals are the most vital orbitals in a molecule and they are considered to characterize the chemical reactivity and kinetic stability. These frontier molecular orbitals are known as the highest occupied molecular orbital (HOMO) and the lowest unoccupied molecular orbital (LUMO) (Table 2). The electronic absorption relates to the transition from the ground to the first excited state and mainly described by one electron excitation from HOMO to LUMO [33]. Kinetic stability increases with the increase of HOMO-LUMO gap. As a result, removal of electrons from ground state HOMO to excited state LUMO requires more energy.

Table 2. Energy (eV) of HOMO, LUMO, gap, hardness and softness of methyl $\alpha$ D-glucopyranoside and its derivatives.

\begin{tabular}{cccccc}
\hline Entry & ${ }^{\boldsymbol{\varepsilon}}$ HOMO & ${ }^{\boldsymbol{\varepsilon}}$ LUMO & Gap & Hardness $(\boldsymbol{\eta})$ & Softness $(\mathbf{S})$ \\
\hline 1 & -6.1918 & 1.3761 & 7.5679 & 3.7839 & 0.2643 \\
2 & -8.4740 & -4.5011 & 3.9729 & 1.9864 & 0.5034 \\
3 & -8.4953 & -4.4935 & 4.0018 & 2.0009 & 0.4997 \\
4 & -6.5738 & -0.8288 & 5.7450 & 2.8725 & 0.3481 \\
5 & -6.5643 & -2.3802 & 4.1841 & 2.0920 & 0.4780 \\
6 & -6.6206 & -0.3828 & 6.2378 & 3.1189 & 0.3206 \\
7 & -6.5398 & -0.3238 & 6.2160 & 3.1080 & 0.3217 \\
8 & -6.5110 & -0.3213 & 6.1897 & 3.0948 & 0.3231 \\
9 & -8.4525 & -5.1153 & 3.3372 & 1.6686 & 0.5993 \\
10 & -8.4939 & -4.9400 & 3.5539 & 1.7769 & 0.5627 \\
11 & -8.5372 & -5.5452 & 2.9920 & 1.4960 & 0.6684 \\
12 & -8.4944 & -5.3934 & 3.1010 & 1.5505 & 0.6449 \\
\hline
\end{tabular}


70 Thermochemical, Molecular Docking and ADMET Studies of Some Methyl $\alpha$-D-Glucopyranoside Derivatives

Table 2 presents the values of orbital energies, along with the two global chemical descriptors, hardness and softness, which were calculated for all compounds. The highest softness was observed for compound $\mathbf{1 1}$ which also showed the lowest HOMO-LUMO gap $(2.9920 \mathrm{eV})$ and hardness, indicating that the molecule is more reactive than other compounds according to Pearson et al [34,35]. On the other hand, compound 6 displayed the highest HOMO-LUMO gap $(6.2378 \mathrm{eV})$, which was less than the parent compound $\mathbf{1}(7.5619 \mathrm{eV})$ which indicates that derivative $\mathbf{1 1}$ had stabilityclose to that of methyl $\alpha$-D-glucopyranoside (1). In Figure 4, the LUMO plot of compound $\mathbf{6}$ showed that the electrons were localized at the modified acylating groups regions only, whereas the HOMO plot indicated that the electrons were localized on the upper part of the pyranose ring. Figure 5 presents the DOS plot for the highest and lowest energy gaps of the modified derivatives.

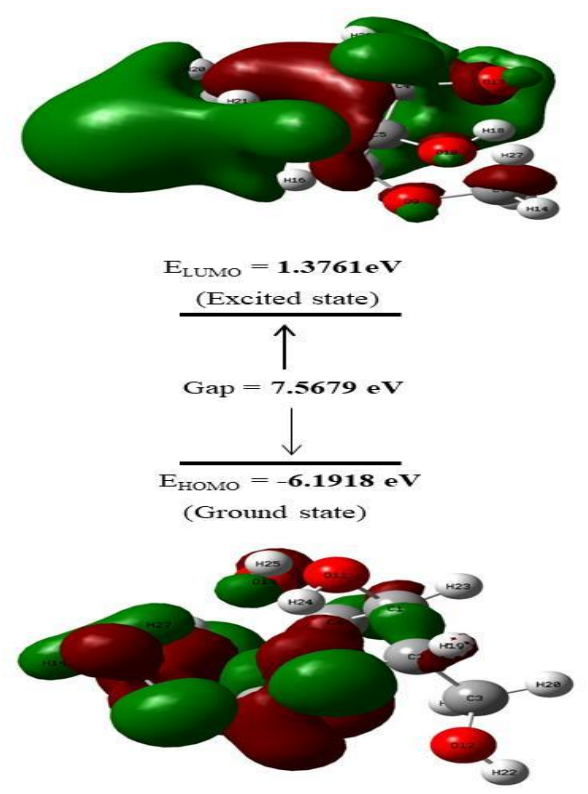

Methyl $\alpha$-D-galactopyranoside (1)

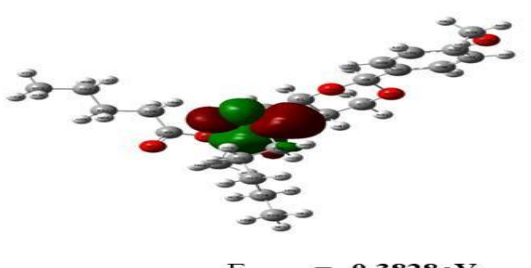

$\mathrm{E}_{\text {IUMO }}=-\mathbf{0 . 3 8 2 8 \mathrm { eV }}$ (Excited state)
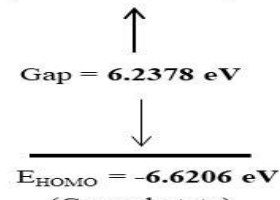

(Ground state)

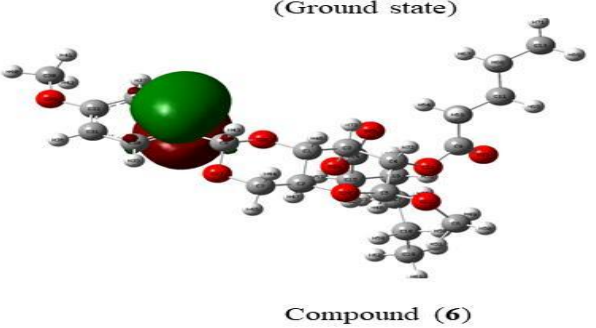

Figure 4. Molecular orbital distribution plots of HOMO and LUMO of methyl $\alpha$ D-glucopyranoside (1) and its derivative $\mathbf{6}$. 

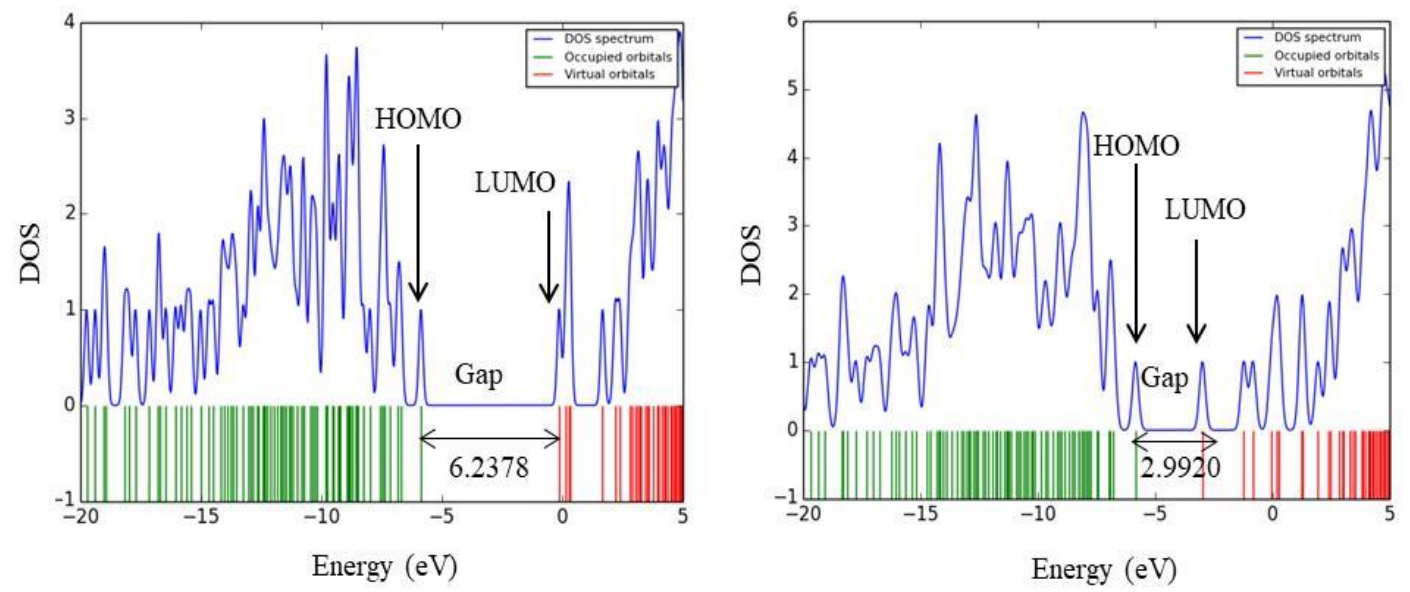

Figure 5. DOS plot and HOMO-LUMO energy gap of derivatives $\mathbf{6}$ (left) and $\mathbf{1 1}$ (right).

\subsection{Molecular electrostatic potential (MEP) study}

In computer-aided drug design, the atomic charges are employed to investigate the connectivity between structure and biological activity of drug. The MEP is worldwide used as reactivity map displaying most suitable region for the electrophilic and nucleophilic attack of charged point like reagents on organic molecules [36]. It helps to interpret biological recognition process and hydrogen bonding interaction [37]. MEP counter map provides a simple way to predict how different geometry could interact. The MEP of title compound is obtained based on the B3LYP with basis set 3-21G optimized result and shown in Figure 6. The importance of MEP lies in the fact that it simultaneously shows a molecular size, shape as well as positive, negative and neutral electrostatic potential regions in terms of color grading and very useful in research of molecular structure with physicochemical properties relationship [38]. Molecular electrostatic potential was calculated to forecast the reactive sites for electrophilic and nucleophilic attack of optimized structure of methyl $\alpha$-D-glucopyranoside (1) and its derivatives (7, 11 and 12). The different values of electrostatic potential represents by different 
72 Thermochemical, Molecular Docking and ADMET Studies of Some Methyl $\alpha$-D-Glucopyranoside Derivatives

colors. Potential increases in the order red $<$ orange $<$ yellow $<$ green $<$ blue. Red color displays maximum negative area which shows favorable site for electrophilic attack, blue color indicate the maximum positive area favorable for nucleophilic attack and green color represent zero potential area.
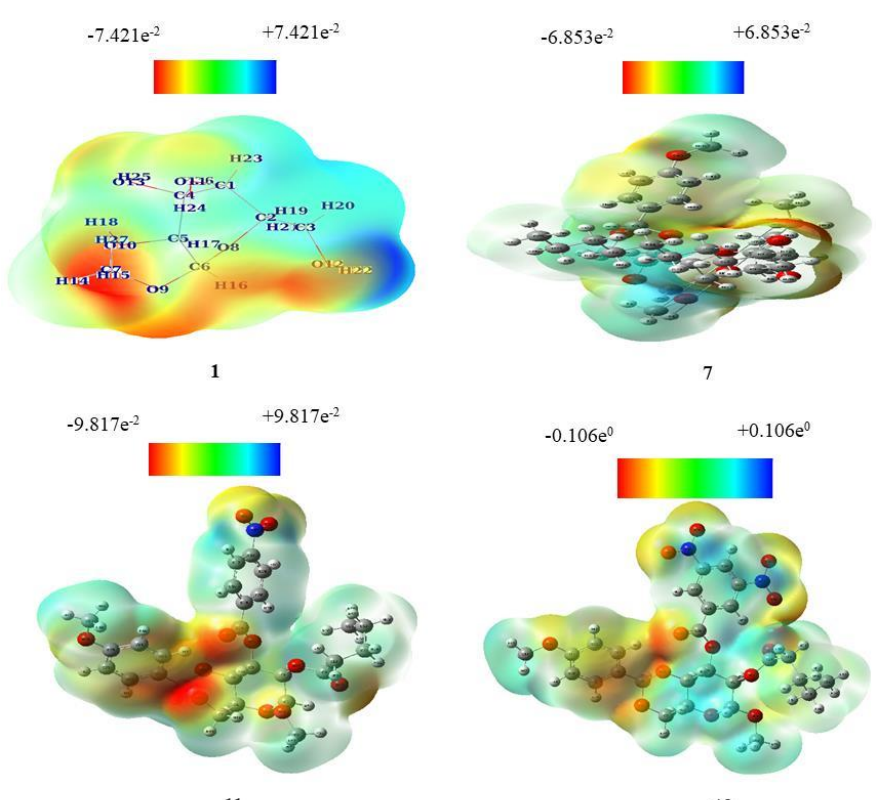

11

12

Figure 6. Molecular electrostatic potential map of compounds (1, 7, 11 and 12).

\subsection{Molecular docking analysis}

Molecular docking is one of the most common methods used in structure based drug design to analyze the interaction between a small molecule and a protein at the atomic level. Molecular docking was performed for those compounds $(\mathbf{1}, \mathbf{3}, \mathbf{9}, \mathbf{1 0}$, 11 and 12), which showed better biological (antibacterial and antifungal) activity by Autodock Vina to investigate their binding mode (Table 3). Afterward, all designated derivatives were docked into the same binding pocket of (PDB ID. 
1LNZ) using similar optimized docking conditions to investigate their binding mode. The outcomes of the docking analysis showed that all compounds, along with the parent compound, obtain binding affinities ranging from -5.3 to -6.8 $\mathrm{kcal} / \mathrm{mol}$. As shown in Table 3, all derivatives exhibited higher binding affinities compared to the parent compound $\mathbf{1}$. These studies revealed that modification of $\mathrm{OH}$ group along with a long carbon chain/aromatic ring molecule increased the binding affinity, while the addition of hetero groups like $-\mathrm{Cl}$ and $-\mathrm{NO}_{2}$ made some fluctuations in binding affinities; however, modification with halogenated aromatic rings increased the binding affinity. The docked conformation clearly showed that the drugs molecules bind within the active site of the Bacillus subtilis Obg (PDB: 1LNZ) macromolecular structure (Figure 7). Figure 8 showed that compound 1 and 3 binds firmly through conventional hydrogen bonds with residues Arg236, Glu50, Arg238, Lys156 and Glu333 besides other interactions such as pi-sigma Arg236 (electrostatic) and pi-cation Pro91 (hydrophobic). But the closer distance was observed for Arg236 (1.93125 ̊). Chlorinated derivative 10 having binding affinity $-6.3 \mathrm{kcal} / \mathrm{mol}$ showed some similar binding site with residues Pro91 and Lys 156 with new hydrophobic interaction, pi alkyl but it also exhibited two different interaction with the residues Arg232 (shorter distance $2.11348 \AA$ ) and Leu158. On the other hand, compound 9 which has a lower binding affinity (-5.6 $\mathrm{kcal} / \mathrm{mol}$ ) than the compound $\mathbf{1 0}$, showed a different binding site due to the structural difference of $-\mathrm{Cl}$ atom. It showed pi-alkyl interaction with Lys40 and Arg121 and conventional hydrogen bond with His78 and Arg128 (shorter distance $2.55176 \AA$ ). It is clear from the structural contrast compound $(\mathbf{9}, \mathbf{1 0}, 11$ and 12) has an additional aromatic (halogenated ring and nitrobenzene ring) substituent in the parent structure, providing a high density of electron in the molecule leading to comparatively higher binding affinity (-5.6, -6.3, -6.4 and -6.8$) \mathrm{kcal} / \mathrm{mol}$. Compound 11 showed three similar binding sites as like as previous derivatives with Arg238, Pro91 and Glu333 within new types of interaction pi-donor hydrogen bond and pi-sigma. 
74 Thermochemical, Molecular Docking and ADMET Studies of Some Methyl $\alpha$-D-Glucopyranoside Derivatives

Table 3. Binding affinity $(\mathrm{kcal} / \mathrm{mol})$ and nonbonding interactions of methyl $\alpha$-Dglucopyranoside and its derivatives.

\begin{tabular}{|c|c|c|c|c|c|c|}
\hline Entry & Protein & $\begin{array}{l}\text { Binding } \\
\text { affinity }\end{array}$ & $\begin{array}{c}\text { Bond } \\
\text { category }\end{array}$ & $\begin{array}{l}\text { Residues } \\
\text { in contact }\end{array}$ & $\begin{array}{c}\text { Interaction } \\
\text { types }\end{array}$ & $\begin{array}{l}\text { Distance } \\
\text { (̊̊) }\end{array}$ \\
\hline \multirow[t]{5}{*}{1} & 1LNZ & -5.3 & Hydrogen & ARG236 & $\mathrm{H}$ & 2.3534 \\
\hline & & & Hydrogen & ARG236 & $\mathrm{H}$ & 1.93125 \\
\hline & & & Hydrogen & ARG238 & $\mathrm{H}$ & 2.45165 \\
\hline & & & Hydrogen & ARG238 & $\mathrm{H}$ & 2.63419 \\
\hline & & & Hydrogen & GLU50 & $\mathrm{C}$ & 3.14971 \\
\hline \multirow[t]{7}{*}{3} & $1 \mathrm{LNZ}$ & -5.6 & Hydrogen & GLU333 & $\mathrm{H}$ & 2.10389 \\
\hline & & & Hydrogen & ARG53 & $\mathrm{H}$ & 2.38636 \\
\hline & & & Hydrogen & ARG236 & $\mathrm{H}$ & 2.68142 \\
\hline & & & Hydrogen & GLU50 & $\mathrm{C}$ & 3.17114 \\
\hline & & & Hydrogen & LYS156 & $\mathrm{C}$ & 3.62682 \\
\hline & & & Electrostatic & ARG236 & PS & 3.62664 \\
\hline & & & Hydrophobic & PRO91 & $\mathrm{PCa}$ & 3.60523 \\
\hline \multirow[t]{5}{*}{9} & 1LNZ & -5.6 & Hydrogen & ARG128 & $\mathrm{H}$ & 2.76541 \\
\hline & & & Hydrogen & ARG128 & $\mathrm{H}$ & 2.55176 \\
\hline & & & Hydrogen & HIS78 & $\mathrm{C}$ & 3.1407 \\
\hline & & & Hydrophobic & LYS40 & PA & 4.06248 \\
\hline & & & Hydrophobic & ARG121 & PA & 4.95581 \\
\hline \multirow[t]{8}{*}{10} & $1 \mathrm{LNZ}$ & 6.3 & Hydrogen & LYS156 & $\mathrm{H}$ & 2.76572 \\
\hline & & & Hydrogen & ARG232 & $\mathrm{H}$ & 2.11348 \\
\hline & & & Hydrogen & ARG236 & $\mathrm{H}$ & 2.49138 \\
\hline & & & Electrostatic & ARG236 & $\mathrm{PCa}$ & 3.22617 \\
\hline & & & Hydrophobic & ARG232 & A & 3.88312 \\
\hline & & & Hydrophobic & LYS156 & PA & 3.95929 \\
\hline & & & Hydrophobic & LEU158 & PA & 5.20024 \\
\hline & & & Hydrophobic & PRO91 & PA & 4.89608 \\
\hline
\end{tabular}




\begin{tabular}{ccccccc}
\hline Entry & Protein & $\begin{array}{c}\text { Binding } \\
\text { affinity }\end{array}$ & $\begin{array}{c}\text { Bond } \\
\text { category }\end{array}$ & $\begin{array}{c}\text { Residues } \\
\text { in contact }\end{array}$ & $\begin{array}{c}\text { Interaction } \\
\text { types }\end{array}$ & $\begin{array}{c}\text { Distance } \\
(\AA)\end{array}$ \\
\hline 11 & 1LNZ & -6.4 & Hydrogen & ARG53 & H & 2.4282 \\
& & & Hydrogen & ARG53 & H & 2.44807 \\
& & Hydrogen & ARG238 & H & 2.61262 \\
& & & Hydrogen & ARG238: & H & 2.4633 \\
& & & Hydrogen & GLY51 & C & 3.7278 \\
& & Hydrogen & GLU333 & PDH & 3.15384 \\
& & & Hydrophobic & GLU333 & PS & 3.97714 \\
& & & Hydrophobic & PRO91 & A & 4.50075 \\
& & & Hydrophobic & PRO332 & PA & 4.99767 \\
12 & \multirow{4}{*}{ 1LNZ } & \multirow{2}{*}{6.8} & Hydrogen & LYS171 & H & 2.24138 \\
& & & Hydrogen & TYR268 & H & 2.09897 \\
& & Hydrogen & ASP212 & C & 3.37442 \\
& & & Hydrophobic & PRO167 & A & 4.44366 \\
& & & Hydrophobic & PRO214 & PA & 5.15455 \\
& & & Hydrophobic & PRO195 & PA & 5.01739 \\
\hline
\end{tabular}

$\mathrm{H}=$ Conventional hydrogen bond; $\mathrm{C}=$ Carbon hydrogen bond; $\mathrm{A}=\mathrm{Alkyl} ; \mathrm{PA}=\mathrm{Pi}-\mathrm{Alkyl}$; $\mathrm{PCa}=$ Pi-Cation; $\mathrm{PDH}=\mathrm{Pi}$-Donor hydrogen bond; PS = Pi-Sigma.

However, this compound firmly interacts with the residue Arg53 at a shorter distance $(2.4282 \AA)$. Finally, compound $\mathbf{1 2}$ which have the highest binding affinity showed some diverse binding site with the residues Lys171, Asp212 and Thr268 (shorter distance $2.09897 \AA$ ). Moreover, these derivatives showed alkyl and pialkyl interaction with Pro167, Pro214 and Pro195. Binding affinity and binding specialty are increased in order of $(3<9<10<11<12)$ due to significant hydrogen bonding. It was observed that modifications of $-\mathrm{OH}$ group of methyl $\alpha$-Dglucopyranoside 1 increased the $\pi$ - $\pi$ interactions with the residues of the active site while increasing their polarity resulted in the formation of hydrogen bonding 
76 Thermochemical, Molecular Docking and ADMET Studies of Some Methyl $\alpha$-D-Glucopyranoside Derivatives

interactions. The maximum numbers of H-bonds were obtained for the compound 11, forming with Arg53, Arg238, Gly51 and Glu333 residues.

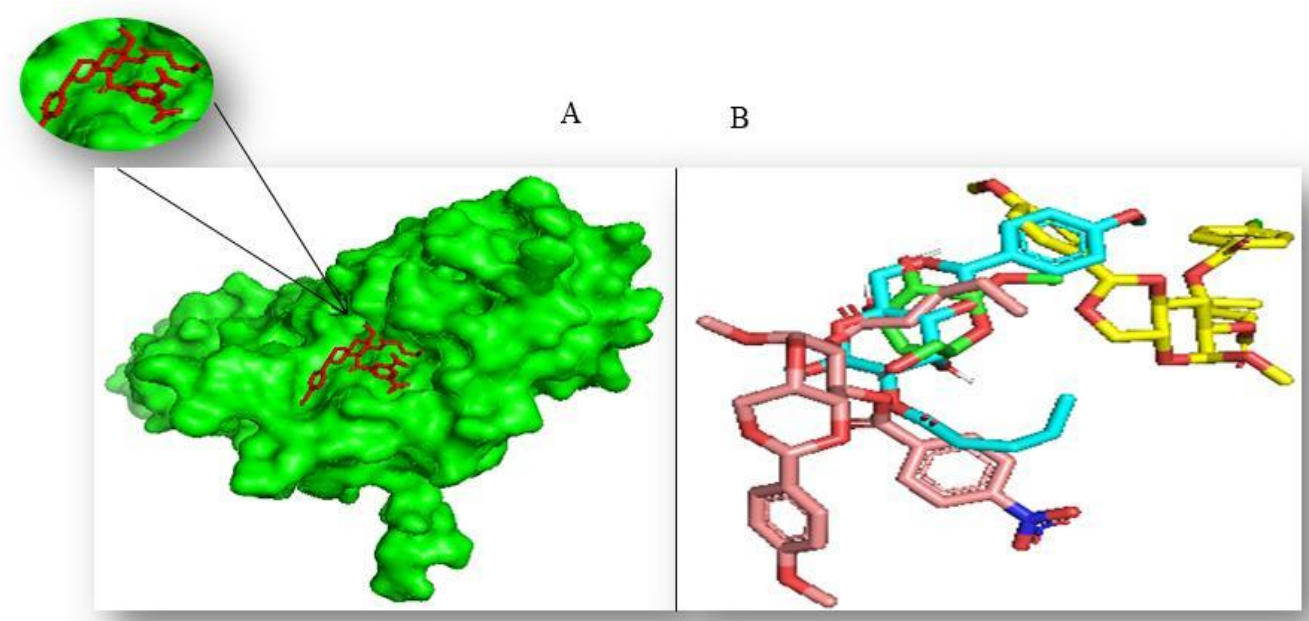

Figure 7. Docked conformation of compound 12 at inhibition bounding site of 1LNZ (A) and superimposed view of all compounds after rigid docking (B). 


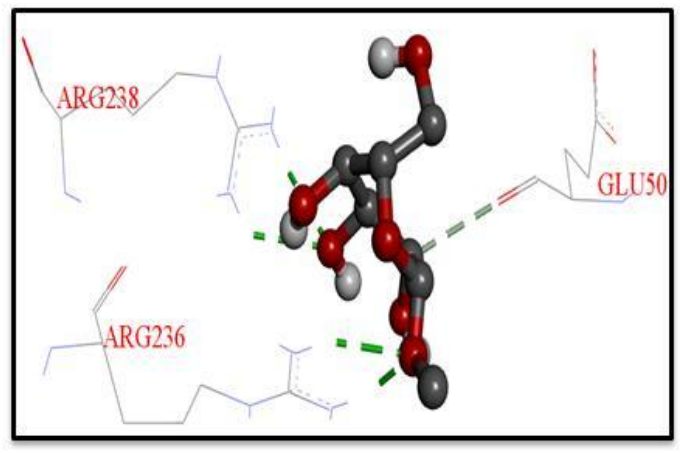

Methyl $\alpha$-D-galactopyranoside (1)

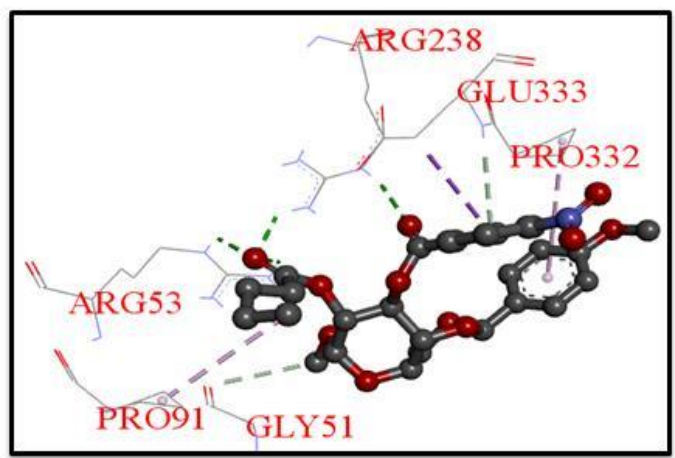

Compound (11)

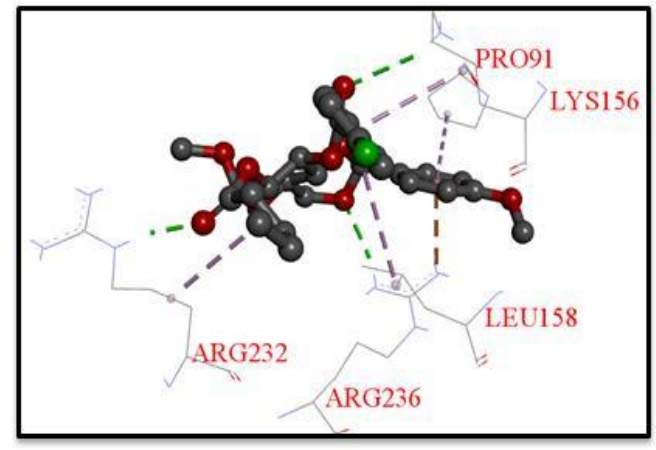

Compound (10)

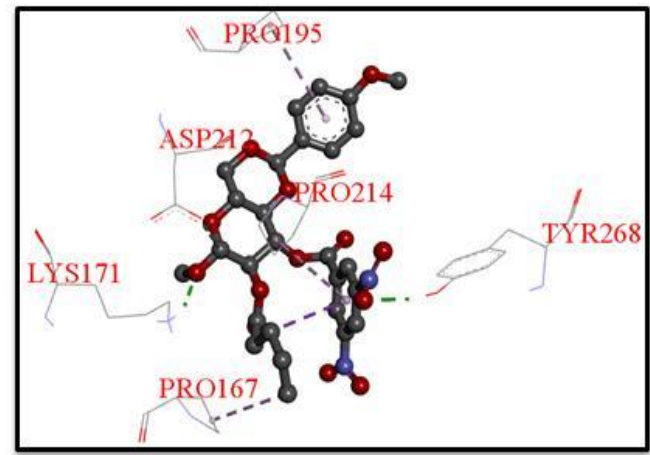

Compound (12)

Figure 8. Non-bonding interactions of compounds (1, 10, 11 and 12) with the amino acid residues of 1LNZ made by Discovery Studio.

In contrast, compounds $\mathbf{1 ,} \mathbf{3}$ and $\mathbf{1 0}$ formed similar numbers H-bonds with the same residues except for Arg236, Glu50 and Lys156. Again, Compound 12 showed characteristic H-bond interaction with residues Lys171, Tyr268 and Asp212, despite having different bonding distances. Hydrogen-bonds execute a vital function in shaping the specificity of ligand binding with the receptor, drug design in chemical and biological processes, molecular recognition and biological activity [39]. The hydrogen bond surface and hydrophobic surface of compound 11 
78 Thermochemical, Molecular Docking and ADMET Studies of Some Methyl $\alpha$-D-Glucopyranoside Derivatives

consequently represent in Figure 9. A hydrophobic surface indicates a partial representation of the molecules based on hydrophobic groups should improve the quality of the results in finding molecular recognition sites, as compared to full representation. As the compound $\mathbf{1 1}$ showed the highest numbers of hydrogen type non-bonding interaction but it has a significant range of hydrophobicity due to having a heteroaromatic ring. We realize that the analyzed bind within the active site of the 1LNZ, the receptor protein of Bacillus subtilis Obg, which is inevitable to prevent the bacterial function. The calculated binding affinities varied in the range of $(-5.3$ to $-6.8 \mathrm{kcal} / \mathrm{mol})$ suggesting the molecules can spontaneously interact within the binding site of Bacillus subtilis Obg. Among all the molecules, the inhibition activity of the compound $\mathbf{1 2}$ was found to be the highest $(-6.8 \mathrm{kcal} / \mathrm{mol})$. Based on the results, we were able to predict the possible binding conformation of the activated form of Obg with the active site. So, it may suggest that these methyl $\alpha$-D-glucopyranoside (1) derivatives may be a potential drug for the inhibition of stress activation of a transcription factor that is caused by Obg.
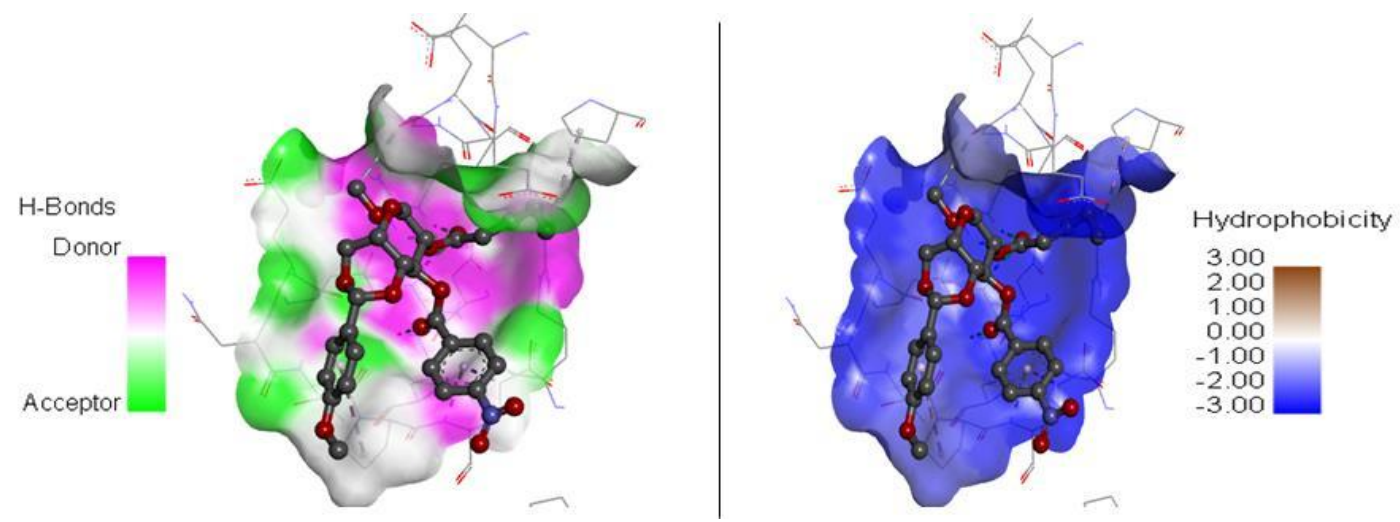

Figure 9. Hydrogen bond and hydrophobic surface of 1LNZ with compound 11. 


\subsection{Pharmacokinetic analysis}

To aid in a drug designing program, accurate data on pharmacokinetics and metabolism must be established as early as possible as it eventually contributes to the final success or failure of the compound. The beginning of early ADMET screening has dramatically decreased the proportion of compounds failing in clinical trials. The prime objective of preclinical ADMET is to eliminate weak drug candidates in the early stages of drug development which allow resources to be focused on potential drug candidates. Pharmacokinetic properties have been predicted to evaluate the absorption, metabolism, and toxicity of all methyl $\alpha$-D-

Table 4. Pharmacokinetic properties of methyl $\alpha$-D-glucopyranoside and its derivatives.

\begin{tabular}{ccccccc}
\hline Entry & BBB & $\begin{array}{c}\text { Human } \\
\text { intestinal } \\
\text { absorption }\end{array}$ & $\begin{array}{c}\text { P- } \\
\text { glycoprotein } \\
\text { inhibitor }\end{array}$ & hERG & Carcinogen & $\begin{array}{c}\text { Acute } \\
\text { oral } \\
\text { toxicity }\end{array}$ \\
\hline 1 & $+(0.8193)$ & $+(0.9782)$ & $\mathrm{NI}(0.9612)$ & $\mathrm{WI}(0.5371)$ & $\mathrm{NC}(0.9857)$ & $\mathrm{III}$ \\
2 & $-(0.5766)$ & $-(0.6252)$ & $\mathrm{NI}(0.8737)$ & $\mathrm{WI}(0.3877)$ & $\mathrm{NC}(0.9571)$ & $\mathrm{III}$ \\
3 & $+(08431)$ & $+(0.7281)$ & $\mathrm{NI}(0.6417)$ & $\mathrm{WI}(0.3891)$ & $\mathrm{NC}(0.9714)$ & $\mathrm{III}$ \\
4 & $+(0.9246)$ & $+(0.8364)$ & $\mathrm{NI}(0.8543)$ & $\mathrm{WI}(0.7883)$ & $\mathrm{NC}(0.9714)$ & $\mathrm{III}$ \\
5 & $+(0.9730)$ & $+(0.8089)$ & $\mathrm{NI}(0.8089)$ & $\mathrm{WI}(0.7438)$ & $\mathrm{NC}(0.8286)$ & $\mathrm{III}$ \\
6 & $+(0.9243)$ & $+(0.8458)$ & $\mathrm{NI}(0.8086)$ & $\mathrm{WI}(07914)$ & $\mathrm{NC}(0.9857)$ & $\mathrm{III}$ \\
7 & $+(0.9343)$ & $+(0.8458)$ & $\mathrm{NI}(0.8204)$ & $\mathrm{WI}(0.8807)$ & $\mathrm{NC}(0.9857)$ & $\mathrm{III}$ \\
8 & $+(0.9343)$ & $+(0.8458)$ & $\mathrm{NI}(0.7813)$ & $\mathrm{WI}(0.8007)$ & $\mathrm{NC}(0.9857)$ & $\mathrm{III}$ \\
9 & $+(0.9596)$ & $+(0.9459)$ & $\mathrm{NI}(0.8788)$ & $\mathrm{WI}(0.8989)$ & $\mathrm{NC}(0.9429)$ & $\mathrm{III}$ \\
10 & $+(0.9596)$ & $+(0.9459)$ & $\mathrm{NI}(0.8917$ & $\mathrm{WI}(0.9099)$ & $\mathrm{NC}(0.9429)$ & $\mathrm{III}$ \\
11 & $+(0.9683)$ & $+(0.8691)$ & $\mathrm{NI}(0.8444)$ & $\mathrm{WI}(0.6969)$ & $\mathrm{NC}(0.9143)$ & $\mathrm{III}$ \\
12 & $+(0.9697)$ & $+(0.8691)$ & $\mathrm{NI}(0.7866)$ & $\mathrm{WI}(0.6586)$ & $\mathrm{NC}(0.9143)$ & $\mathrm{III}$ \\
\hline$+=$ Positive, NI $=$ Non-inhibitor, WI $=\mathrm{Weak}$ inhibitor, NC $=\mathrm{Non}-\mathrm{carcinogenic}, \mathrm{III}=$ \\
Category III includes compounds with LD50 greater than 500 mg/kg but less than 5000 \\
mg/kg.
\end{tabular}


80 Thermochemical, Molecular Docking and ADMET Studies of Some Methyl $\alpha$-D-Glucopyranoside Derivatives

glucopyranoside derivatives. AdmetSAR calculation (Table 4) predicts these methyl $\alpha$-D-glucopyranoside derivatives are non-carcinogenic and possess category III oral toxicity, so methyl $\alpha$-D-glucopyranoside derivatives may be suggested to be relatively harmless for oral administration. All drugs are P-glycoprotein noninhibitor where P-glycoprotein inhibitor can interrupt the absorption, permeability and retention of the drugs. All compounds exhibit positivity allowing for the bloodbrain barrier (BBB). However, all the monosaccharide derivatives have shown weak inhibitory characteristics for human ether -a go-go-Related Gene (hERG). Inhibitory feature of hERG can lead to long QT syndrome [40], that's why further investigation is required on this aspect.

\section{Conclusion}

In this study, the implicit characteristic stability and biochemical behavior of methyl $\alpha$-D-glucopyranoside and its synthesized derivatives are investigated by the quantum mechanical method. The most significant properties for biological chemistry, chemical reactivity and frontier orbital study like HOMO, LUMO, HOMO-LUMO gap, and molecular electrostatic potential in molecule were optimized which indicates that it may be good drug molecules. Most of the designed methyl $\alpha$-D-glucopyranoside derivatives have a HOMO-LUMO gap closer to the parent ligand $\mathbf{1}$ and have an exalted pharmacokinetic profile than the parent ligand. So, it may be concluded that some modified compounds were more reactive than the parent drug, as they showed lower HOMO-LUMO energy gaps and some may stable like methyl $\alpha$-D-glucopyranoside (1). Molecular docking analysis statedthe docked poses of derivatives $(\mathbf{3}, \mathbf{9}, \mathbf{1 0}, \mathbf{1 1}$, and 12) with $1 \mathrm{LNZ}$ showed a better binding affinity with significant nonbonding interactions than the parent molecule. These blind molecular docking studies suggest a potential approach for the application of antibacterials drugs as expected inhibitors of Bacillus subtilis Obg which may reduce the stress activation of a transcription factor. The molecular electrostatic potential study also showed the most negative and positive surface area of the investigated ligand and hence anticipated the 
suitable site for the hydrogen bonding site. The pharmacokinetic study suggested that the modified derivatives were less toxic and have improved pharmacokinetic properties than the parent drug. Finally, this research may be useful to understand the chemical, thermal,physicochemical, biological and pharmacokinetic properties of methyl $\alpha$-D-glucopyranoside derivatives.

\section{Acknowledgement}

The authors are grateful to the Research and Publication Cell, University of Chittagong, Bangladesh for providing financial support to carry out this research project.

\section{References}

[1] C.R. Bertozzi and L. L. Kiessling: Science, 2001, 291(5512), 2357.

[2] S. Chen and M. Fukuda: Methods in Enzymology, 2006, 416, 371.

[3] A. Varki: Glycobiology, 1993, 3(2), 97.

[4] P. H. Seeberger and D.B. Werz:Nature, 2007, 446(7139), 1046.

[5] R. Gupta, S. Paul, A.K. Gupta, P. L. Kachroo and S. Bani: Indian Journal of Chemistry, 1997, 36, 707.

[6] R. Sing, B. B Gupta, O.M. P. Mallik and H. R. Kataria: Pesticide Science, 1987, 20, 125 .

[7] S. M. A. Kawsar, M. O. Faruk, M. S. Rahman, Y. Fujii and Y. Ozeki: Scientia Pharmaceutica, 2014, 82(1), 1.

[8] S. M. A. Kawsar, A. K. M. S. Kabir, M. M. R. Bhuiyan and M. M. Manik: Journal of the Bangladesh Chemical Society, 2012, 25(2), 101.

[9] S. M. A. Kawsar, A. K. M. S. Kabir, M. M. Manik, M. K. Hossain and M. N. Anwar: International Journal of Biosciences, 2012, 2(7), 66.

[10] A.K.M.S. Kabir, S.M.A. Kawsar, M.M.R. Bhuiyan, M.S. Rahman and M.E. Chowdhury:Pakistan Journal of Scientific and Industrial Research, 2009,52(3), 138.

[11] M. M. H. Misbah, J. Ferdous, M. Z. H. Bulbul, T. S. Chowdhury, S. Dey, I. Hasan and S. M. A. Kawsar: International Journal of Biosciences, 2020, 16(4), 299. 
82 Thermochemical, Molecular Docking and ADMET Studies of Some Methyl $\alpha$-D-Glucopyranoside Derivatives

[12] A.K.M.S. Kabir, P. Dutta and M.N. Anwar:International Journal of Agriculture and Biology,2005, 7(5), 760.

[13] M. M. Islam, M. Arifuzzaman, M. M. Rahman, M. A. Rahman and S. M. A. Kawsar:Hacettepe Journal of Biology and Chemistry, 2019, 47(2), 153.

[14] S. M. A. Kawsar,M. M. Rahman, M. Islam, M. A. Manchur, I. Hasan and S. Rajia: International Journal of Biosciences, 2018, 12(6), 408.

[15] S. M. A. Kawsar, M. A. Hosen, Y.Fujii and Y.Ozeki:Journal of Computational Chemistry \& Molecular Modeling, 2020,4(4), 452.

[16] S. M. A. Kawsar, M. A. Hosen:Turkish Computational and Theoretical Chemistry, 2020,4(2), 59.

[17] M. Z. H. Bulbul, M. A. Hosen, J.Ferdous, T. S. Chowdhury, M. M.H. Misbah and S. M. A. Kawsar: International Journal of New Chemistry, 2021, 8(1), 88.

[18] M. S. Janelle, J. U. Jingliang, T. Mitchell andW. G. Haldenwang:Journal ofBacteriology, 2000,182(10), 2771.

[19] R. A.Gaussian09, M. J.Frisch, G. W.Trucks, H. B.Schlegel, G. E.Scuseria, M. A.Robb, J. R.Cheeseman, G.Scalmani, V.Barone, B.Mennucci, G. A.Petersson et al, Gaussian, Inc, Wallingford CT., USA, 2009. https://gaussian.com/g09citation/

[20] A. D. Becke:Physical Review A, 1988,38(6), 3098.

[21] C. Lee, W. Yang and R. G. Parr:Physical Review B, 1988,37(2), 785.

[22] R. G. Pearson:Proceedings of the National Academy of Sciences, 1986, 83(22), 8440 .

[23] H. M. Berman, J. Westbrook, Z. Feng, G. Gilliland, T. N. Bhat and H. Weissig:Nucleic Acids Research,2000, 28(1), 235.

[24] Delano wl, The PyMOL Molecular Graphics System. De-Lano Scientific, San Carlos, CA, USA, 2002.

[25] N. Guex and M. C. Peitsch:Electrophoresis,1997, 18(15), 2714.

[26] S. Dallakyan, A. J. Olson, Small-Molecule Library Screening by Docking with Py Rx. In: Hempel JE, Williams CH, Hong CC (Eds.). Chemical Biology: Methods and Protocols. Springer New York, USA, 243, 2015.

[27] Version ADS 4.0, Accelrys, San Diego, USA, 2017.

[28] F.Cheng, W.Li, Y.Zhou, J.Shen, Z.Wu, G.Liu, P. W. Lee and Y. Tang: Journal of Chemical Information and Modeling, 2012,52(11), 3099. 
[29] C. A. Lipinski, F. Lombardo, B.W. Dominy and P.J. Feeney:Advanced Drug Delivery Reviews, 2001, 46(1-3), 3.

[30] S. M. A. Kawsar, A. K. M. S.Kabir, M. M. R.Bhuiyan, J. FerdousandM. S.Rahman: Journal of Bangladesh Academy of Sciences, 2013, 37(2), 145.

[31] N. Cohen and S. W. Benson:Chemical Reviews, 1993,93(7) 2419.

[32] E. J. Lien, Z. R. Guo, R. L. Li and C. T. Su:Journal of Pharmaceutical Sciences, 1982,71(6), 641.

[33] S.Saravanan and V.Balachandran:Spectrochimicaacta. Part A, Molecular and Biomolecular Spectroscopy,2014, 120, 351.

[34] M. M. Hoque, M. A. Halim, M. G. Sarwar andM. Khan:Journal of Physical Organic Chemistry, 2015, 28, 732.

[35] R. G. Parr and Z. Zhou: Accounts of Chemical Research, 1993, 26(5), 256.

[36] M. L. Amin: Drug Target Insights, 2013, 2013(7), 27.

[37] P. Politzer and J. S. Murray: Reviews in Computational Chemistry, 1991, 2, 273.

[38] P. Politzer, D. G. Truhlar (Eds.), Chemical Applications of Atomic and Molecular Electrostatic Potentials, Plenum Press, NY, USA, 1981.

https://10.1007/978-1-4757-9634-6

[39] D. Gautam and S. Thomas, "The Weak Hydrogen Bond In Structural Chemistry and Biology", Oxford University Press, UK, 2010. https://10.1093/acprof:oso/9780198509707.001.0001

[40] M. C. Sanguinetti and M. T. Firouz: Nature, 2006, 440(7083), 463.

The Chittagong Univ. J. Sc. Vol. 42(1), 2020 